\title{
DIFFERENCE SEQUENCE OF FIBONACCI $t$ NUMBERS
}

\author{
Eunmi Choi \\ Department of Mathematics \\ HanNam University \\ Daejeon, KOREA
}

\begin{abstract}
We study interrelationships of Fibonacci $s$ and Fibonacci $t$ numbers for any $s \neq t$. Let $d_{n}^{(s, t)}$ be the difference sequence of Fibonacci $s$ and $t$ numbers. We investigate recurrence formulas of $d_{n}^{(s, t)}$ and find patterns that both Fibonacci $s$ and Fibonacci $t$ numbers satisfy at the same time. Moreover we research increasing ratio of the sequence $d_{n}^{(s, t)}$.
\end{abstract}

AMS Subject Classification: 11B30, 11B37

Key Words: Fibonacci $t$ number, difference sequence of Fibonacci numbers

\section{Introduction}

For any integer $t>0$, a Fibonacci $t$ number $f_{n}^{(t)}$ satisfies the recurrence $f_{n+1}^{(t)}=$ $f_{n}^{(t)}+f_{n-t}^{(t)}$ with $t+1$ initials $1, \cdots, 1,2$. If $t=1$ then it is the ordinary Fibonacci number and if $t=2$ then it is sometimes called a Narayana number, see [4]. Fibonacci $t$ numbers have many analogous properties of Fibonacci numbers, like Binet formula, sums of numbers and associated matrices, etc (see [2], [3], or [5]). In particular the limit of ratio of consecutive Fibonacci $t$ numbers satisfies the equation $P_{t}(x)=x^{t+1}-x^{t}-1$ (see [6]), and its application to to data-hiding was discussed in [1].

Though Fibonacci $t$ numbers were studied in various aspect, it seems no one has asked interrelationships between Fibonacci $t$ and Fibonacci $s$ numbers for $t \neq s$. In this work we define a difference sequence $d_{n}^{(s, t)}$ of Fibonacci $s$

$\begin{array}{lr}\text { Received: } & \text { August 19, } 2016 \\ \text { Revised: } & \text { September 19, } 2016 \\ \text { Published: } & \text { November 4, 2016 }\end{array}$

(C) 2016 Academic Publications, Ltd. url: www.acadpubl.eu 
and Fibonacci $t$ numbers. We investigate recurrence formulas of $d_{n}^{(s, t)}$, and find a recurrence pattern that both Fibonacci $s$ and Fibonacci $t$ numbers satisfy, and moreover examine increasing ratios of difference sequences $d_{n}^{(s, t)}$. Since $f_{n}^{(1)}$ is well known, Fibonacci $t$ numbers $f_{n}^{(t)}$ would be obtained from $d_{n}^{(1, t)}$. In particular we discuss about $d_{n}^{(1,4)}$ that has rather unique property.

\section{Difference Sequence of $f_{n}^{(1)}$ and $f_{n}^{(t)}$}

The Fibonacci $t$ number $f_{n}^{(t)}$ starts from $1, \cdots, 1,2$ and satisfies the recurrence $f_{n+1}^{(t)}=f_{n}^{(t)}+f_{n-t}^{(t)}$ for $n-t>0$. The $f_{n}^{(t)}$ can be extended to negative parameter $n$ so that we have the following table of $f_{n}^{(t)}$ for $1 \leq t \leq 4$ :

\begin{tabular}{r|rrrrrrrrrrrrrr}
$n$ & -6 & -5 & -4 & -3 & -2 & -1 & 0 & 1 & 2 & 3 & 4 & 5 & 6 & 7 \\
\hline$f_{n}^{(1)}$ & 5 & -3 & 2 & -1 & 1 & 0 & 1 & 1 & 2 & 3 & 5 & 8 & 13 & 21 \\
$f_{n}^{(2)}$ & -1 & 1 & 0 & 0 & 1 & 0 & 1 & 1 & 1 & 2 & 3 & 4 & 6 & 9 \\
$f_{n}^{(3)}$ & 0 & 0 & 1 & 0 & 0 & 0 & 1 & 1 & 1 & 1 & 2 & 3 & 4 & 5 \\
$f_{n}^{(4)}$ & 0 & 1 & 0 & 0 & 0 & 0 & 1 & 1 & 1 & 1 & 1 & 2 & 3 & 4
\end{tabular}

The next lemma explore some identities on $f_{n}^{(t)}(1 \leq t \leq 3)$.

Lemma 1. For any $t \geq 1$, we have $f_{n+1}^{(t)}=\sum_{i=t-1}^{2 t-1} f_{n-i}^{(t)}$. Moreover we have the following equalities:

(1) $f_{n}^{(1)}=4 f_{n-3}^{(1)}+f_{n-6}^{(1)}, 3 f_{n}^{(1)}=4 f_{n-1}^{(1)}+f_{n-2}^{(1)}+f_{n-4}^{(1)}$.

(2) $f_{n}^{(2)}+2 f_{n-2}^{(2)}+f_{n-4}^{(2)}=f_{n+2}^{(2)}, f_{n}^{(3)}=4 f_{n-5}^{(3)}+3 f_{n-8}^{(3)}-f_{n-9}^{(3)}+f_{n-11}^{(3)}$.

(3) $f_{n}^{(3)}=2 f_{n-2}^{(3)}-f_{n-11}^{(3)}-f_{n-12}^{(3)}, f_{n}^{(3)}=2 f_{n-1}^{(3)}-f_{n-4}^{(3)}-f_{n-5}^{(3)}+f_{n-13}^{(3)}+f_{n-14}^{(3)}$.

Proof. Clearly: $f_{n+1}^{(1)}=\sum_{i=0}^{1} f_{n-i}^{(1)}, f_{n+1}^{(2)}=\left(f_{n-1}^{(2)}+f_{n-3}^{(2)}\right)+f_{n-2}^{(2)}=\sum_{i=1}^{3} f_{n-i}^{(2)}$ and $f_{n+1}^{(3)}=\left(f_{n-1}^{(3)}+f_{n-4}^{(3)}\right)+f_{n-3}^{(2)}=\left(f_{n-2}^{(3)}+f_{n-5}^{(3)}\right)+f_{n-4}^{(3)}+f_{n-3}^{(2)}=\sum_{i=2}^{5} f_{n-i}^{(3)}$.

Now for $t \geq 1$, consider $\sum_{i=t-1}^{2 t-1} f_{n-i}^{(t)}=f_{n-(t-1)}^{(t)}+\cdots+f_{n-(2 t-1)}^{(t)}$. Then by adding the last term to the first one, we have

$$
\sum_{i=t-1}^{2 t-1} f_{n-i}^{(t)}=\left[f_{n-(t-1)}^{(t)}+f_{n-(2 t-1)}^{(t)}\right]+f_{n-t}^{(t)}+f_{n-(t+1)}^{(t)}+\cdots+f_{n-(2 t-2)}^{(t)}
$$




$$
=f_{n-(t-2)}^{(t)}+f_{n-t}^{(t)}+f_{n-(t+1)}^{(t)}+\cdots+f_{n-(2 t-3)}^{(t)}+f_{n-(2 t-2)}^{(t)} .
$$

Continuing to add the last to the first term, it follows that

$$
\begin{aligned}
\sum_{i=t-1}^{2 t-1} f_{n-i}^{(t)} & =f_{n-(t-3)}^{(t)}+f_{n-t}^{(t)}+f_{n-(t+1)}^{(t)} \\
& =\cdots \\
& =f_{n-1}^{(t)}+f_{n-t}^{(t)}+f_{n-t-1}^{(t)} \\
& =f_{n}^{(t)}+f_{n-t}^{(t)} \\
& =f_{n+1}^{(t)} .
\end{aligned}
$$

Now the identities on $f_{n}^{(1)}$ are clear, and we have

$$
f_{n}^{(2)}+2 f_{n-2}^{(2)}+f_{n-4}^{(2)}=f_{n+1}^{(2)}+f_{n-1}^{(2)}=f_{n+2}^{(2)} .
$$

For Fibonacci 3 numbers, it follows that

$$
\begin{aligned}
f_{n}^{(3)} & =f_{n-3}^{(3)}+f_{n-4}^{(3)}+f_{n-5}^{(3)}+f_{n-6}^{(3)} \\
& =2 f_{n-4}^{(3)}+f_{n-5}^{(3)}+f_{n-6}^{(3)}+f_{n-7}^{(3)} \\
& =3 f_{n-5}^{(3)}+f_{n-6}^{(3)}+f_{n-7}^{(3)}+2 f_{n-8}^{(3)}
\end{aligned}
$$

so that

$$
\begin{aligned}
f_{n}^{(3)}-4 f_{n-5}^{(3)} & =-f_{n-5}^{(3)}+f_{n-6}^{(3)}+f_{n-7}^{(3)}+2 f_{n-8}^{(3)} \\
& =-\left(f_{n-6}^{(3)}+f_{n-9}^{(3)}\right)+f_{n-6}^{(3)}+f_{n-7}^{(3)}+2 f_{n-8}^{(3)} \\
& =3 f_{n-8}^{(3)}-f_{n-9}^{(3)}+f_{n-11}^{(3)} .
\end{aligned}
$$

Moreover we also have

$$
\begin{aligned}
f_{n}^{(3)}-f_{n-2}^{(3)}+f_{n-11}^{(3)}+f_{n-12}^{(3)}= & \left(f_{n-1}^{(3)}+f_{n-4}^{(3)}\right)-f_{n-2}^{(3)}+\left(f_{n-7}^{(3)}\right. \\
& \left.-f_{n-8}^{(3)}\right)+\left(f_{n-8}^{(3)}-f_{n-9}^{(3)}\right) \\
= & f_{n-1}^{(3)}-f_{n-2}^{(3)}+f_{n-4}^{(3)}+f_{n-7}^{(3)}-\left(f_{n-5}^{(3)}-f_{n-6}^{(3)}\right) \\
= & {\left[f_{n-1}^{(3)}-f_{n-2}^{(3)}-f_{n-5}^{(3)}\right]+f_{n-4}^{(3)}+f_{n-6}^{(3)}+f_{n-7}^{(3)} } \\
= & f_{n-3}^{(3)}+f_{n-6}^{(3)} \\
= & f_{n-2}^{(3)},
\end{aligned}
$$


since $f_{n-1}^{(3)}-f_{n-2}^{(3)}-f_{n-5}^{(3)}=0$. Finally it is not hard to see that

$2 f_{n-1}^{(3)}-f_{n-4}^{(3)}-f_{n-5}^{(3)}+f_{n-13}^{(3)}+f_{n-14}^{(3)}=f_{n-1}^{(3)}+\left(f_{n-2}^{(3)}-f_{n-4}^{(3)}+f_{n-13}^{(3)}+f_{n-14}^{(3)}\right)$.

But since $f_{n-2}^{(3)}=2 f_{n-4}^{(3)}-f_{n-13}^{(3)}-f_{n-14}^{(3)}$, it follows immediately that

$$
2 f_{n-1}^{(3)}-f_{n-4}^{(3)}-f_{n-5}^{(3)}+f_{n-13}^{(3)}+f_{n-14}^{(3)}=f_{n-1}^{(3)}+f_{n-4}^{(3)}=f_{n}^{(3)} .
$$

For any $n, i \geq 0$, let $d_{n,(i)}^{(s, t)}=f_{n}^{(s)}-f_{n+i}^{(t)}$ and call $\left\{d_{n,(i)}^{(s, t)}\right\}$ the $i$ step difference sequence of Fibonacci $s$ and $t$ numbers. In particular when $i=0$, we denote the difference sequence by $\left\{d_{n,(i)}^{(s, t)}\right\}=\left\{d_{n}^{(s, t)}\right\}$. We begin to study recurrence formula of the difference sequence $d_{n,(i)}^{(1,2)}=f_{n}^{(1)}-f_{n+i}^{(2)}$.

Theorem 2. $d_{n,(i)}^{(1,2)}$ satisfies

$$
d_{n+1,(i)}^{(1,2)}=2 d_{n,(i)}^{(1,2)}-d_{n-3,(i)}^{(1,2)}-d_{n-4,(i)}^{(1,2)},
$$

for all $i \geq 0$.

Proof. For convenience, write $d_{n,(0)}^{(1,2)}=d_{n}$. Then $\left\{d_{n}\right\}_{n \geq 1}=\{0,1,1,2,4$, $7,12, \cdots\}$, so if $n=5$ then $2 d_{5}-d_{2}-d_{1}=7=d_{6}$. Suppose the recurrence is true for all $k<n$. Then the induction hypothesis shows

$$
\begin{aligned}
2 d_{n}-d_{n-3}-d_{n-4}= & 2\left(2 d_{n-1}-d_{n-4}-d_{n-5}\right)-\left(2 d_{n-4}-d_{n-7}-d_{n-8}\right) \\
& -\left(2 d_{n-5}-d_{n-8}-d_{n-9}\right) \\
= & 4 d_{n-1}-4 d_{n-4}-4 d_{n-5}+d_{n-7}+2 d_{n-8}+d_{n-9} .
\end{aligned}
$$

So by substituting $d_{n}^{(1,2)}=f_{n}^{(1)}-f_{n}^{(2)}$, we have

$$
2 d_{n}-d_{n-3}-d_{n-4}=A^{(1)}-A^{(2)},
$$

where

$$
A^{(j)}=4 f_{n-1}^{(j)}-4 f_{n-4}^{(j)}-4 f_{n-5}^{(j)}+f_{n-7}^{(j)}+2 f_{n-8}^{(j)}+f_{n-9}^{(j)},
$$

for $j=1,2$.

It is not hard to see from Lemma 1 that

$$
\begin{aligned}
A^{(1)} & =4 f_{n-1}^{(1)}-4\left(f_{n-4}^{(1)}+f_{n-5}^{(1)}\right)+\left(f_{n-7}^{(1)}+f_{n-8}^{(1)}\right)+\left(f_{n-8}^{(1)}+f_{n-9}^{(1)}\right) \\
& =4 f_{n-2}^{(1)}+f_{n-6}^{(1)}+f_{n-7}^{(1)} \\
& =f_{n+1}^{(1)}-f_{n-5}^{(1)}+f_{n-6}^{(1)}+f_{n-7}^{(1)}=f_{n+1}^{(1)} .
\end{aligned}
$$


And Lemma 1 also shows

$$
\begin{aligned}
A^{(2)} & =4 f_{n-1}^{(2)}-4 f_{n-4}^{(2)}-4 f_{n-5}^{(2)}+f_{n-7}^{(2)}+2 f_{n-8}^{(2)}+f_{n-9}^{(2)} \\
& =4\left(f_{n-1}^{(2)}-f_{n-4}^{(2)}-f_{n-5}^{(2)}\right)+\left(f_{n-7}^{(2)}+f_{n-8}^{(2)}+f_{n-9}^{(2)}\right)+f_{n-8}^{(2)} \\
& =4 f_{n-3}^{(2)}+f_{n-5}^{(2)}+\left(f_{n-4}^{(2)}-f_{n-6}^{(2)}-f_{n-7}^{(2)}\right) \\
& =\left(f_{n-3}^{(2)}+f_{n-4}^{(2)}+f_{n-5}^{(2)}\right)+3 f_{n-3}^{(2)}-f_{n-6}^{(2)}-f_{n-7}^{(2)} \\
& =f_{n-1}^{(2)}+2 f_{n-3}^{(2)}+\left(f_{n-3}^{(2)}-f_{n-6}^{(2)}-f_{n-7}^{(2)}\right) \\
& =f_{n+1}^{(2)} .
\end{aligned}
$$

Therefore we have $2 d_{n}-d_{n-3}-d_{n-4}=f_{n+1}^{(1)}-f_{n+1}^{(2)}=d_{n+1}$.

Now write $d_{n,(i)}^{(1,2)}=d_{n,(i)}$ for convenience.

If $i=1$, then

$$
\left\{d_{n,(1)}=f_{n}^{(1)}-f_{n+1}^{(2)}\right\}=\{0,0,0,1,2,4,8,15,27,48, \cdots\}
$$

and hence $d_{n+1,(1)}=2 d_{n,(1)}-d_{n-3,(1)}-d_{n-4,(1)}$ is true for $n \leq 10$.

Assume the identity is true for all $k<n$. Then

$$
\begin{aligned}
2 d_{n,(1)}-d_{n-3,(1)}-d_{n-4,(1)}= & 4 d_{n-1,(1)}-4 d_{n-4,(1)}-4 d_{n-5,(1)}+d_{n-7,(1)} \\
& +2 d_{n-8,(1)}+d_{n-9,(1)} \\
= & \left(4 f_{n-1}^{(1)}-4 f_{n-4}^{(1)}-4 f_{n-5}^{(1)}+f_{n-7}^{(1)}+2 f_{n-8}^{(1)}+f_{n-9}^{(1)}\right) \\
& -\left(4 f_{n}^{(2)}-4 f_{n-3}^{(2)}-4 f_{n-4}^{(2)}+f_{n-6}^{(2)}+2 f_{n-7}^{(2)}+f_{n-8}^{(2)}\right) .
\end{aligned}
$$

As seen above, it is easy to see that

$$
4 f_{n-1}^{(1)}-4 f_{n-4}^{(1)}-4 f_{n-5}^{(1)}+f_{n-7}^{(1)}+2 f_{n-8}^{(1)}+f_{n-9}^{(1)}=f_{n+1}^{(1)}
$$

and

$$
4 f_{n}^{(2)}-4 f_{n-3}^{(2)}-4 f_{n-4}^{(2)}+f_{n-6}^{(2)}+2 f_{n-7}^{(2)}+f_{n-8}^{(2)}=f_{n+2}^{(2)},
$$

hence it follows that

$$
2 d_{n,(1)}-d_{n-3,(1)}-d_{n-4,(1)}=f_{n+1}^{(1)}-f_{n+2}^{(2)}=d_{n+1,(1)} .
$$

Now for any $i \geq 0$, we also have

$$
\begin{aligned}
2 d_{n,(i)}-d_{n-3,(i)}-d_{n-4,(i)}= & 4 d_{n-1,(i)}-4 d_{n-4,(i)}-4 d_{n-5,(i)} \\
& +d_{n-7,(i)}+2 d_{n-8,(i)}+d_{n-9,(i)} \\
= & f_{n+1}^{(1)}-f_{n+1+i}^{(2)}=d_{n+1,(i)} .
\end{aligned}
$$

This completes the proof. 
Theorem 2 gives a relation that both $f_{n}^{(1)}$ and $f_{n}^{(2)}$ satisfy.

Corollary 3. $f_{n}^{(j)}(j=1,2)$ satisfies $f_{n+1}^{(j)}=2 f_{n}^{(j)}-f_{n-3}^{(j)}-f_{n-4}^{(j)}$.

Proof. The identity is clear for $f_{n}^{(1)}$. And since $f_{n}^{(2)}=f_{n-2}^{(2)}+f_{n-3}^{(2)}+f_{n-4}^{(2)}$ by Lemma 1 , we have

$$
f_{n+1}^{(2)}=f_{n}^{(2)}+f_{n-2}^{(2)}=2 f_{n}^{(2)}-f_{n-3}^{(2)}-f_{n-4}^{(2)} \text {. }
$$

Theorem 4. The difference $d_{n,(i)}^{(1,3)}=f_{n}^{(1)}-f_{n+i}^{(3)}$ satisfies $d_{n+1,(i)}^{(1,3)}=2 d_{n,(i)}^{(1,3)}-$ $d_{n-2,(i)}^{(1,3)}+d_{n-3,(i)}^{(1,3)}-d_{n-4,(i)}^{(1,3)}-d_{n-5,(i)}^{(1,3)}$.

Proof. Let $i=0$ and $d_{n,(0)}^{(1,3)}=d_{n}$. Then $\left\{d_{n}\right\}_{n \geq 1}=\{0,1,2,3,5,9,16,27, \cdots\}$ shows $2 d_{6}-d_{4}+d_{3}-d_{2}-d_{1}=16=d_{7}$. Hence if we assume the identity is true for all $k<n$ then similar to the proof of Theorem 2, we have

$$
\begin{aligned}
2 d_{n}-d_{n-2}+d_{n-3}-d_{n-4}-d_{n-5}= & 4 d_{n-1}-4 d_{n-3}+4 d_{n-4}-3 d_{n-5}-6 d_{n-6} \\
& +3 d_{n-7}+d_{n-10}-\left(d_{n-9}-d_{n-10}-d_{n-11}\right) .
\end{aligned}
$$

So due to the induction hypothesis $d_{n-5}=2 d_{n-6}-d_{n-8}+d_{n-9}-d_{n-10}-d_{n-11}$, we have

$$
\begin{aligned}
2 d_{n}-d_{n-2}+d_{n-3}-d_{n-4}-d_{n-5}= & 4 d_{n-1}-4 d_{n-3}+4 d_{n-4}-3 d_{n-5}-6 d_{n-6} \\
& +3 d_{n-7}+d_{n-10}-\left(d_{n-5}-2 d_{n-6}+d_{n-8}\right) \\
= & 4 d_{n-1}-4 d_{n-3}+4 d_{n-4}-4 d_{n-5} \\
& -4 d_{n-6}+3 d_{n-7}-d_{n-8}+d_{n-10} .
\end{aligned}
$$

Now substitute $d_{n}=f_{n}^{(1)}-f_{n}^{(3)}$. Then it follows that $2 d_{n}-d_{n-2}+d_{n-3}-d_{n-4}-$ $d_{n-5}=A^{(1)}-A^{(3)}$ where,

$$
A^{(j)}=4 f_{n-1}^{(j)}-4 f_{n-3}^{(j)}+4 f_{n-4}^{(j)}-4 f_{n-5}^{(j)}-4 f_{n-6}^{(j)}+3 f_{n-7}^{(j)}-f_{n-8}^{(j)}+f_{n-10}^{(j)}
$$

for $j=1,3$. We shall show $A^{(j)}=f_{n+1}^{(j)}$.

If so, we finish to prove $2 d_{n}-d_{n-2}+d_{n-3}-d_{n-4}-d_{n-5}=f_{n+1}^{(1)}-f_{n+1}^{(3)}=d_{n+1}$.

It is not hard to see

$$
\begin{aligned}
A^{(1)} & =4\left(f_{n-1}^{(1)}-f_{n-3}^{(1)}\right)+4\left(f_{n-4}^{(1)}-f_{n-6}^{(1)}\right)-4\left(f_{n-5}^{(1)}-f_{n-7}^{(1)}\right)-f_{n-7}^{(1)}-\left(f_{n-8}^{(1)}-f_{n-10}^{(1)}\right) \\
& =4 f_{n-2}^{(1)}+\left(4 f_{n-5}^{(1)}-4 f_{n-6}^{(1)}-f_{n-7}^{(1)}-f_{n-9}^{(1)}\right) .
\end{aligned}
$$

But since $3 f_{n}^{(1)}=4 f_{n-1}^{(1)}+f_{n-2}^{(1)}+f_{n-4}^{(1)}$ (Lemma 1$)$, we have $4 f_{n}^{(1)}-4 f_{n-1}^{(1)}-$ $f_{n-2}^{(1)}-f_{n-4}^{(1)}=f_{n}^{(1)}$, hence

$$
A^{(1)}=4 f_{n-2}^{(1)}+f_{n-5}^{(1)}=3 f_{n-2}^{(1)}+2 f_{n-3}^{(1)}=f_{n-2}^{(1)}+2 f_{n-1}^{(1)}=f_{n+1}^{(1)} .
$$


Similarly due to Lemma 1, we also have

$$
\begin{aligned}
A^{(3)}= & 4\left(f_{n-1}^{(3)}+f_{n-4}^{(3)}\right)-4\left(f_{n-3}^{(3)}+f_{n-6}^{(3)}\right)-\left(f_{n-5}^{(3)}+f_{n-8}^{(3)}\right) \\
& -3 f_{n-5}^{(3)}+\left(f_{n-7}^{(3)}+f_{n-10}^{(3)}\right)+2 f_{n-7}^{(3)} \\
= & 4 f_{n}^{(3)}-3\left(f_{n-2}^{(3)}+f_{n-5}^{(3)}\right)-f_{n-2}^{(3)}+2\left(f_{n-4}^{(3)}+2 f_{n-7}^{(3)}\right)-3 f_{n-4}^{(3)}+f_{n-6}^{(3)} \\
= & 4 f_{n}^{(3)}-3\left(f_{n-1}^{(3)}+f_{n-4}^{(3)}\right)-f_{n-2}^{(3)}+\left(f_{n-3}^{(3)}+f_{n-6}^{(3)}\right)+f_{n-3}^{(3)} \\
= & 4 f_{n}^{(3)}-3 f_{n}^{(3)}-f_{n-2}^{(3)}+f_{n-2}^{(3)}+f_{n-3}^{(3)} \\
= & f_{n}^{(3)}+f_{n-3}^{(3)} \\
= & f_{n+1}^{(3)} .
\end{aligned}
$$

Now if $i>0$ then the proof follows similar to Theorem 2 .

The recurrence of $d_{n+1}^{(1,3)}$ yields an identity that both $f_{n}^{(1)}$ and $f_{n}^{(3)}$ hold.

Corollary 5. $f_{n+1}^{(j)}=2 f_{n}^{(j)}-f_{n-2}^{(j)}+f_{n-3}^{(j)}-f_{n-4}^{(j)}-f_{n-5}^{(j)}$ for $j=1,3$.

Proof. The identity for $f_{n}^{(1)}$ is clear. On the other hand, Lemma 1 says $f_{n}^{(3)}=f_{n-3}^{(3)}+f_{n-4}^{(3)}+f_{n-5}^{(3)}+f_{n-6}^{(3)}=f_{n-2}^{(3)}+f_{n-4}^{(3)}+f_{n-5}^{(3)}$, hence we have

$$
\begin{aligned}
& 2 f_{n}^{(3)}-f_{n-2}^{(3)}+f_{n-3}^{(3)}-f_{n-4}^{(3)}-f_{n-5}^{(3)} \\
& \quad=2 f_{n}^{(3)}-f_{n-2}^{(3)}+f_{n-3}^{(3)}-f_{n}^{(3)}+f_{n-2}^{(3)} \\
& \quad=f_{n}^{(3)}+f_{n-3}^{(3)} \\
& \quad=f_{n+1}^{(3)} \cdot \square
\end{aligned}
$$

Besides Theorem 4, another recurrence of $d_{n,(i)}^{(1,3)}$ is as follows.

Theorem 6. $\quad d_{n+1,(i)}^{(1,3)}=2 d_{n,(i)}^{(1,3)}-d_{n-3,(i)}^{(1,3)}-d_{n-4,(i)}^{(1,3)}-f_{n-12}^{(3)}-f_{n-13}^{(3)}$ for all $i \geq 0$. Moreover $f_{n}^{(3)}+f_{n-1}^{(3)}=d_{n+10}^{(1,3)}-2 d_{n+9}^{(1,3)}+d_{n+7}^{(1,3)}$.

Proof. Without loss of generality we may assume $i=0$. Then

$$
\begin{aligned}
& 2 d_{n}^{(1,3)}-d_{n-3}^{(1,3)}-d_{n-4}^{(1,3)}-f_{n-12}^{(3)}-f_{n-13}^{(3)} \\
& \quad=2\left(f_{n}^{(1)}-f_{n}^{(3)}\right)-\left(f_{n-3}^{(1)}-f_{n-3}^{(3)}\right)-\left(f_{n-4}^{(1)}-f_{n-4}^{(3)}\right)-\left(f_{n-12}^{(3)}-f_{n-13}^{(3)}\right) \\
& =\left(2 f_{n}^{(1)}-f_{n-3}^{(1)}-f_{n-4}^{(1)}\right)-\left(2 f_{n}^{(3)}-f_{n-3}^{(3)}-f_{n-4}^{(3)}+f_{n-12}^{(3)}+f_{n-13}^{(3)}\right) \\
& =f_{n+1}^{(1)}-f_{n+1}^{(3)} \\
& =d_{n+1}^{(1,3)}
\end{aligned}
$$


by Lemma 1. Hence together with Theorem 4, it follows that

$$
\begin{aligned}
2 d_{n}^{(1,3)}-d_{n-2}^{(1,3)}+d_{n-3}^{(1,3)}-d_{n-4}^{(1,3)}-d_{n-5}^{(1,3)} & =d_{n+1}^{(1,3)} \\
& =2 d_{n}^{(1,3)}-d_{n-3}^{(1,3)}-d_{n-4}^{(1,3)}-f_{n-12}^{(3)}-f_{n-13}^{(3)},
\end{aligned}
$$

so we have $f_{n-12}^{(3)}+f_{n-13}^{(3)}=d_{n-2}^{(1,3)}-2 d_{n-3}^{(1,3)}+d_{n-5}^{(1,3)}$.

We further study $f_{n}^{(4)}$ and difference sequence $d_{n}^{(1,4)}=f_{n}^{(1)}-f_{n}^{(4)}$.

Theorem 7. $d_{n}^{(1,4)}$ satisfies $d_{n+1}^{(1,4)}=2 d_{n}^{(1,4)}-d_{n-2}^{(1,4)}+d_{n-4}^{(1,4)}-d_{n-5}^{(1,4)}-d_{n-6}^{(1,4)}$.

Proof. We first verify $2 f_{n}^{(j)}-f_{n-2}^{(j)}+f_{n-4}^{(j)}-f_{n-5}^{(j)}-f_{n-6}^{(j)}=f_{n+1}^{(j)}$ for $j=1,4$. If $j=1$ then the identity is clear. If $j=4$ then

$$
\begin{aligned}
2 & f_{n}^{(4)}-f_{n-2}^{(4)}+f_{n-4}^{(4)}-f_{n-5}^{(4)}-f_{n-6}^{(4)} \\
& =f_{n}^{(4)}+\left(f_{n}^{(4)}+f_{n-4}^{(4)}\right)-\left(f_{n-2}^{(4)}+f_{n-6}^{(4)}\right)-f_{n-5}^{(4)} \\
& =f_{n}^{(4)}+f_{n+1}^{(4)}-f_{n-1}^{(4)}-f_{n-5}^{(4)} \\
& =f_{n}^{(4)}+f_{n+1}^{(4)}-f_{n}^{(4)} \\
& =f_{n+1}^{(4)} .
\end{aligned}
$$

It thus follows immediately that

$$
\begin{aligned}
& 2 d_{n}^{(1,4)}-d_{n-2}^{(1,4)}+d_{n-4}^{(1,4)}-d_{n-5}^{(1,4)}-d_{n-6}^{(1,4)} \\
& =\left(2 f_{n}^{(1)}-f_{n-2}^{(1)}+f_{n-4}^{(1)}-f_{n-5}^{(1)}-f_{n-6}^{(1)}\right)-\left(2 f_{n}^{(4)}-f_{n-2}^{(4)}+f_{n-4}^{(4)}-f_{n-5}^{(4)}-f_{n-6}^{(4)}\right) \\
& =f_{n+1}^{(1)}-f_{n+1}^{(4)} \\
& =d_{n+1}^{(1,4)} .
\end{aligned}
$$

\section{Ratio of Difference Sequence}

The ratio $\frac{f_{n+1}^{(t)}}{f_{n}^{(t)}}$ of consecutive Fibonacci $t$ numbers converges to a positive root of $P_{t}(x)=x^{t+1}-x^{t}-1$ for all $t \geq 1$ (see [6]). In this section we shall investigate the limit of $\frac{d_{n+1,(i)}^{(1, t)}}{d_{n,(i)}^{(1, t)}}$ of difference sequence, moreover find their interrelationships with $P_{t}(x)$. Note that since $d_{n,(i)}^{(1, t)}$ satisfies the same recurrence pattern for all $i>0$, we may enough to consider $\frac{d_{n+1}^{(1, t)}}{d_{n}^{(1, t)}}$. 
Theorem 8. Let $\Delta^{(1,2)}(x)=x^{5}-2 x^{4}+x+1$. Then $\lim _{n \rightarrow \infty} \frac{d_{n+1}^{(1,2)}}{d_{n}^{(1,2)}}$ is a real positive root of $\Delta^{(1,2)}=P_{1}(x) P_{2}(x)$. Moreover $\lim _{n \rightarrow \infty} \frac{d_{n+1}^{(1,2)}}{d_{n}^{(1,2)}}=\lim _{n \rightarrow \infty} \frac{f_{n+1}^{(1)}}{f_{n}^{(1)}}$.

Proof. The recurrence of $d_{n}^{(1,2)}$ in Theorem 2 gives rise to

$$
\frac{d_{n+1}^{(1,2)}}{d_{n}^{(1,2)}}=2-\frac{1}{d_{n}^{(1,2)} / d_{n-3}^{(1,2)}}-\frac{1}{d_{n}^{(1,2)} / d_{n-4}^{(1,2)}}
$$

So by letting $\lim _{n \rightarrow \infty} \frac{d_{n+1}^{(1,2)}}{d_{n}^{(1,2)}}=\alpha$, we have $\alpha=2-\frac{1}{\alpha^{3}}-\frac{1}{\alpha^{4}}$ and $\alpha$ is a positive real root of $x^{5}-2 x^{4}+x+1=\Delta^{(1,2)}(x)$. Clearly

$$
\begin{aligned}
\Delta^{(1,2)}(x) & =x^{5}-2 x^{4}+x+1 \\
& =\left(x^{2}-x-1\right)\left(x^{3}-x^{2}-1\right) \\
& =P_{1}(x) P_{2}(x) .
\end{aligned}
$$

So $\alpha$ is one of the roots of $P_{i}(x)(i=1,2)$ where each $P_{i}(x)$ has only one positive real root that equals $\lim _{n \rightarrow \infty} \frac{f_{n+1}^{(1)}}{f_{n}^{(1)}}=1.6180$ or $\lim _{n \rightarrow \infty} \frac{f_{n+1}^{(2)}}{f_{n}^{(2)}}=1.4655$. Note that

$$
\frac{d_{n+1}^{(1,2)}}{d_{n}^{(1,2)}}=\frac{f_{n+1}^{(1)}-f_{n+1}^{(2)}}{f_{n}^{(1)}-f_{n}^{(2)}}=\frac{\left(f_{n+1}^{(1)}-f_{n+1}^{(2)}\right) / f_{n}^{(1)}}{\left(f_{n}^{(1)}-f_{n}^{(2)}\right) / f_{n}^{(1)}}=\frac{\frac{f_{n+1}^{(1)}}{f_{n}^{(1)}}-\frac{1}{f_{n}^{(1)} / f_{n+1}^{(2)}}}{1-\frac{1}{f_{n}^{(1)} / f_{n}^{(2)}}}
$$

But as $n$ gets larger, $f_{n}^{(1)}$ increases much rapidly than $f_{n}^{(2)}$ so that $\lim _{n \rightarrow \infty} \frac{1}{f_{n}^{(1)} / f_{n}^{(2)}}=$ 0 . It thus follows that $\alpha=\lim _{n \rightarrow \infty} \frac{d_{n+1}^{(1,2)}}{d_{n}^{(1,2)}}=\lim _{n \rightarrow \infty} \frac{f_{n+1}^{(1)}}{f_{n}^{(1)}}$.

In fact, $\frac{d_{n+1}^{(1,2)}}{d_{n}^{(1,2)}}$ equals $1.689,1.640,1.625,1.619$, and 1.618 when $n=10,20$, 30, 40, 50. As $n$ goes to infinite, the ratio $\frac{d_{n+1}^{(1,2)}}{d_{n}^{(1,2)}}$ corresponds to $\frac{f_{n+1}^{(1)}}{f_{n}^{(1)}}$.

Theorem 9. Let $\Delta^{(1,3)}(x)=x^{6}-2 x^{5}+x^{3}-x^{2}+x+1$. Then $\alpha=\lim _{n \rightarrow \infty} \frac{d_{n+1}^{(1,3)}}{d_{n}^{(1,3)}}$ is a real root of $\Delta^{(1,3)}(x)=P_{1}(x) P_{3}(x)$. And $\lim _{n \rightarrow \infty} \frac{d_{n+1}^{(1,3)}}{d_{n}^{(1,3)}}=\lim _{n \rightarrow \infty} \frac{f_{n+1}^{(1)}}{f_{n}^{(1)}}$.

Proof. Write $d_{n}^{(1,3)}=d_{n}$. Since

$$
\frac{d_{n+1}}{d_{n}}=2-\frac{1}{d_{n} / d_{n-2}}+\frac{1}{d_{n} / d_{n-3}}-\frac{1}{d_{n} / d_{n-4}}-\frac{1}{d_{n} / d_{n-5}}
$$


by Theorem $4, \alpha=\lim _{n \rightarrow \infty} \frac{d_{n+1}^{(1,3)}}{d_{n}^{(1,3)}}$ satisfies $\alpha^{6}=2 \alpha^{5}-\alpha^{3}+\alpha^{2}-\alpha+1$ so $\alpha$ is a zero of $\Delta^{(1,3)}(x)$. But since $P_{1}(x) P_{3}(x)=\left(x^{2}-x-1\right)\left(x^{4}-x^{3}-1\right)=\Delta^{(1,3)}(x), \alpha$ is a root of either $P_{1}(x)$ or $P_{3}(x)$. Note that

$$
\frac{d_{n+1}^{(1,3)}}{d_{n}^{(1,3)}}=\frac{\left(f_{n+1}^{(1)}-f_{n+1}^{(3)}\right) / f_{n}^{(1)}}{\left(f_{n}^{(1)}-f_{n}^{(3)}\right) / f_{n}^{(1)}}=\frac{\frac{f_{n+1}^{(1)}}{f_{n}^{(1)}}-\frac{1}{f_{n}^{(1)} / f_{n+1}^{(3)}}}{1-\frac{1}{f_{n}^{(1)} / f_{n}^{(3)}}} .
$$

Since $f_{n}^{(1)}$ increases very faster than $f_{n}^{(3)}$ as $n$ gets larger, $\lim _{n \rightarrow \infty} \frac{1}{f_{n}^{(1)} / f_{n}^{(3)}}=0$ so that $\alpha=\lim _{n \rightarrow \infty} \frac{d_{n+1}^{(1,3)}}{d_{n}^{(1,3)}}=\lim _{n \rightarrow \infty} \frac{f_{n+1}^{(1)}}{f_{n}^{(1)}}$.

The previous results on $d_{n}^{(1, t)}(t=2,3,4)$ yield the next theorem.

Theorem 10. For any integer $t>0$, we have the followings:

(1) $d_{n+1}^{(1, t)}=d_{n}^{(1, t)}-d_{n-2}^{(1, t)}+d_{n-t}^{(1, t)}-d_{n-(t+1)}^{(1, t)}-d_{n-(t+2)}^{(1, t)}$.

(2) $\lim _{n \rightarrow \infty} \frac{d_{n+1}^{(1, t)}}{d_{n}^{(1, t)}}$ is a real root of $\Delta^{(1, t)}(x)=x^{t+3}-2 x^{t+2}+x^{t}-x^{2}+x+1$. Moreover $\Delta^{(1, t)}(x)=P_{1}(x) P_{t}(x)$ and $\lim _{n \rightarrow \infty} \frac{d_{n+1}^{(1, t)}}{d_{n}^{(1, t)}}=\lim _{n \rightarrow \infty} \frac{f_{n+1}^{(1)}}{f_{n}^{(1)}}$.

Proof. When $t=2,3$, it is due to Theorem 8 and 9 . When $t=4$, if we write $d_{n}^{(1,4)}=d_{n}$ then

$$
\frac{d_{n+1}}{d_{n}}=2-\frac{1}{d_{n} / d_{n-2}}+\frac{1}{d_{n} / d_{n-4}}-\frac{1}{d_{n} / d_{n-5}}-\frac{1}{d_{n} / d_{n-6}}
$$

by Theorem 7 .

Hence by letting $\alpha=\lim _{n \rightarrow \infty} \frac{d_{n+1}}{d_{n}}, \alpha$ satisfies $\alpha^{7}=2 \alpha^{6}-\alpha^{4}+\alpha^{2}-\alpha-1$.

Consider the polynomial $P_{t}(x)=x^{t+1}-x^{t}-1$. Clearly

$$
\begin{aligned}
P_{1}(x) P_{4}(x) & =\left(x^{2}-x-1\right)\left(x^{5}-x^{4}-1\right) \\
& =x^{7}-2 x^{6}+x^{4}-x^{2}+x+1 \\
& =\Delta^{(1,4)}(x),
\end{aligned}
$$

so $\alpha$ is a real root of either $P_{1}(x)$ or $P_{4}(x)$. But since $f_{n}^{(1)}$ increases rapidly than $f_{n}^{(4)}$ as $n$ gets larger, we have $\lim _{n \rightarrow \infty} \frac{1}{f_{n}^{(1)} / f_{n}^{(4)}}=0$ so that

$$
\lim _{n \rightarrow \infty} \frac{d_{n+1}^{(1,4)}}{d_{n}^{(1,4)}}=\lim _{n \rightarrow \infty} \frac{\frac{f_{n+1}^{(1)}}{f_{n}^{(1)}}-\frac{1}{f_{n}^{(1)} / f_{n+1}^{(4)}}}{1-\frac{1}{f_{n}^{(1)} / f_{n}^{(4)}}}=\lim _{n \rightarrow \infty} \frac{f_{n+1}^{(1)}}{f_{n}^{(1)}}
$$


Now for any $t>0$, let $\Delta^{(1, t)}(x)=P_{1}(x) P_{t}(x)$. Then $\Delta^{(1, t)}(x)=0$ implies $x^{t+3}=2 x^{t+2}-x^{t}+x^{2}-x-1$, so we may claim that $d_{n}^{(1, t)}$ satisfies

$$
d_{n+1}^{(1, t)}=2 d_{n}^{(1, t)}-d_{n-2}^{(1, t)}+d_{n-t}^{(1, t)}-d_{n-t-1}^{(1, t)}-d_{n-t-2}^{(1, t)} .
$$

Indeed when $1 \leq t \leq 5$, it is clear from the next table:

\begin{tabular}{l|l|l}
$t$ & $P_{1}(x) P_{\mathrm{t}}(x)$ & recurrence of $d_{\mathrm{n}}^{(1, \mathrm{t})}=d_{\mathrm{n}}$ \\
\hline 2 & $x^{5}-2 x^{4}+x+1$ & $d_{\mathrm{n}+1}=2 d_{\mathrm{n}}-d_{\mathrm{n}-3}-d_{\mathrm{n}-4}$ \\
3 & $x^{6}-2 x^{5}+x^{3}-x^{2}+x+1$ & $d_{\mathrm{n}+1}=2 d_{\mathrm{n}}-d_{\mathrm{n}-2}+d_{\mathrm{n}-3}-d_{\mathrm{n}-4}-d_{\mathrm{n}-5}$ \\
4 & $x^{7}-2 x^{6}+x^{4}-x^{2}+x+1$ & $d_{\mathrm{n}+1}=2 d_{\mathrm{n}}-d_{\mathrm{n}-2}+d_{\mathrm{n}-4}-d_{\mathrm{n}-5}-d_{\mathrm{n}-6}$ \\
5 & $x^{8}-2 x^{7}+x^{5}-x^{2}+x+1$ & $d_{\mathrm{n}+1}=2 d_{\mathrm{n}}-d_{\mathrm{n}-2}+d_{\mathrm{n}-5}-d_{\mathrm{n}-6}-d_{\mathrm{n}-7}$
\end{tabular}

Now for any $t>0$, write $d_{n}^{(1, t)}=f_{n}^{(1)}-f_{n}^{(t)}$ by $d_{n}$ for convenience. Then

$$
\begin{aligned}
2 d_{n} & -d_{n-2}+d_{n-t}-d_{n-t-1}-d_{n-t-2} \\
= & \left(2 f_{n}^{(1)}-f_{n-2}^{(1)}+f_{n-t}^{(1)}-f_{n-t-1}^{(1)}-f_{n-t-2}^{(1)}\right) \\
& -\left(2 f_{n}^{(t)}-f_{n-2}^{(t)}+f_{n-t}^{(t)}-f_{n-t-1}^{(t)}-f_{n-t-2}^{(t)}\right) \\
= & f_{n+1}^{(1)}-f_{n+1}^{(t)} \\
= & d_{n+1} .
\end{aligned}
$$

Moreover since $\lim _{n \rightarrow \infty} \frac{1}{f_{n}^{(1)} / f_{n}^{(t)}}=0$, we finish to prove

$$
\lim _{n \rightarrow \infty} \frac{d_{n+1}}{d_{n}}=\lim _{n \rightarrow \infty} \frac{\frac{f_{n+1}^{(1)}}{f_{n}^{(1)}}-\frac{1}{f_{n}^{(1)} / f_{n+1}^{(t)}}}{1-\frac{1}{f_{n}^{(1)} / f_{n}^{(t)}}}=\lim _{n \rightarrow \infty} \frac{f_{n+1}^{(1)}}{f_{n}^{(1)}}
$$

\section{Fibonacci 4 Numbers}

We now give our special attention to $f_{n}^{(4)}$ and $d_{n}^{(1,4)}$, and will prove that for any $n, f_{n}^{(4)}+f_{n-1}^{(4)}-f_{n-2}^{(4)}-2 f_{n-3}^{(4)}-f_{n-4}^{(4)}$ is equal to one of $\pm 1, \pm 2$. Let us define a constant $C_{n}$ that equals

$$
\left\{\begin{array} { l l } 
{ 1 } & { \text { if } n \equiv 0 , 2 } \\
{ 2 } & { \text { if } n \equiv 1 }
\end{array} \text { and } \quad \left\{\begin{array}{ll}
-1 & \text { if } n \equiv 3,5 \\
-2 & \text { if } n \equiv 4
\end{array}\right.\right.
$$

for $n(\bmod 6)$. 
Theorem 11. For any integer $n>0$, we have the followings:

(1) $f_{n}^{(4)}+f_{n-1}^{(4)}-f_{n-2}^{(4)}-2 f_{n-3}^{(4)}-f_{n-4}^{(4)}=C_{n}$.

(2) $f_{n+1}^{(4)}-4 f_{n-1}^{(4)}+4 f_{n-3}^{(4)}-f_{n-5}^{(4)}+f_{n-8}^{(4)}+2 f_{n-9}^{(4)}-f_{n-11}^{(4)}=-2 C_{n}$.

Proof. When $5 \leq n \leq 10$, the next table proves the theorem.

\begin{tabular}{c|rc||r|rr}
$n$ & $C_{\mathrm{n}}$ & $f_{\mathrm{n}}^{(4)}+f_{\mathrm{n}-1}^{(4)}-f_{\mathrm{n}-2}^{(4)}-2 f_{\mathrm{n}-3}^{(4)}-f_{\mathrm{n}-4}^{(4)}$ & $n$ & $C_{\mathrm{n}}$ & \\
\hline 5 & -1 & $2+1-1-2-1=-1$ & 8 & 1 & $5+4-3-4-1=1$ \\
6 & 1 & $3+2-1-2-1=1$ & 9 & -1 & $6+5-4-6-2=-1$ \\
7 & 2 & $4+3-2-2-1=2$ & 10 & -2 & $8+6-5-8-3=-2$
\end{tabular}

First of all, we can observe $C_{n}=C_{n-1}+C_{n-5}(n \bmod 6)$. In fact, if $n \equiv 0$ $(\bmod 6)$ then $C_{n-1}+C_{n-5}=-1+2=1=C_{n}$, if $n \equiv 1$ then $1+1=2=C_{n}$, and so on. We now assume the identity $f_{k}^{(4)}+f_{k-1}^{(4)}=f_{k-2}^{(4)}+2 f_{k-3}^{(4)}+f_{k-4}^{(4)}+C_{k}$ is true for all $k<n$. Then the induction hypothesis implies that

$$
\begin{aligned}
f_{n}^{(4)}+f_{n-1}^{(4)}= & \left(f_{n-1}^{(4)}+f_{n-2}^{(4)}\right)+\left(f_{n-5}^{(4)}+f_{n-6}^{(4)}\right) \\
= & \left(f_{n-3}^{(4)}+2 f_{n-4}^{(4)}+f_{n-5}^{(4)}+C_{n-1}\right) \\
& +\left(f_{n-7}^{(4)}+2 f_{n-8}^{(4)}+f_{n-9}^{(4)}+C_{n-5}\right) \\
= & \left(f_{n-3}^{(4)}+f_{n-7}^{(4)}\right)+2\left(f_{n-4}^{(4)}+f_{n-8}^{(4)}\right)+\left(f_{n-5}^{(4)}+f_{n-9}^{(4)}\right)+\left(C_{n-1}+C_{n-5}\right) \\
= & f_{n-2}^{(4)}+2 f_{n-3}^{(4)}+f_{n-4}^{(4)}+C_{n},
\end{aligned}
$$

so we have (1) that $f_{n}^{(4)}+f_{n-1}^{(4)}-f_{n-2}^{(4)}-2 f_{n-3}^{(4)}-f_{n-4}^{(4)}=C_{n}$ for all $n(\bmod 6)$.

Moreover we also have

$$
\begin{aligned}
& 4 f_{n-1}^{(4)}-4 f_{n-3}^{(4)}+f_{n-5}^{(4)}-f_{n-8}^{(4)}-2 f_{n-9}^{(4)}+f_{n-11}^{(4)} \\
& \quad=\left(f_{n-1}^{(4)}+f_{n-5}^{(4)}\right)+3 f_{n-1}^{(4)}-4 f_{n-3}^{(4)}-f_{n-8}^{(4)}-2 f_{n-9}^{(4)}+f_{n-11}^{(4)} \\
& \quad=f_{n}^{(4)}+3 f_{n-1}^{(4)}-4 f_{n-3}^{(4)}+f_{n-4}^{(4)}-\left(f_{n-4}^{(4)}+f_{n-8}^{(4)}\right)-2 f_{n-9}^{(4)}+f_{n-11}^{(4)} \\
& =\left(f_{n}^{(4)}+f_{n-4}^{(4)}\right)+3 f_{n-1}^{(4)}-5 f_{n-3}^{(4)}-2 f_{n-9}^{(4)}+f_{n-11}^{(4)} \\
& \quad=f_{n+1}^{(4)}+3 f_{n-1}^{(4)}-5 f_{n-3}^{(4)}-2 f_{n-9}^{(4)}+f_{n-11}^{(4)} .
\end{aligned}
$$

But since

$$
f_{n-11}^{(4)}=f_{n-6}^{(4)}-f_{n-7}^{(4)}=f_{n-1}^{(4)}-f_{n-2}^{(4)}-f_{n-7}^{(4)}
$$


we have

$$
\begin{aligned}
& f_{n+1}^{(4)}-4 f_{n-1}^{(4)}+4 f_{n-3}^{(4)}-f_{n-5}^{(4)}+f_{n-8}^{(4)}+2 f_{n-9}^{(4)}-f_{n-11}^{(4)} \\
& \quad=f_{n+1}^{(4)}-f_{n+1}^{(4)}-3 f_{n-1}^{(4)}+5 f_{n-3}^{(4)}+2 f_{n-4}^{(4)}-2 f_{n-5}^{(4)}-f_{n-1}^{(4)}+f_{n-2}^{(4)}+f_{n-7}^{(4)} \\
& =-2\left(f_{n-1}^{(4)}+f_{n-5}^{(4)}\right)-2 f_{n-1}^{(4)}+f_{n-2}^{(4)}+\left(f_{n-3}^{(4)}+f_{n-7}^{(4)}\right)+4 f_{n-3}^{(4)}+2 f_{n-4}^{(4)} \\
& =-2\left(f_{n}^{(4)}+f_{n-1}^{(4)}-f_{n-2}^{(4)}-2 f_{n-3}^{(4)}-f_{n-4}^{(4)}\right) \\
& =-2 C_{n} .
\end{aligned}
$$

Theorem 11 gives rise to a more simple recurrence of $d_{n}^{(1,4)}$.

Theorem 12. Let $\lambda_{n}=1$ (if $n \equiv 0,1$ ), 0 (if $n \equiv 2,5$ ) and -1 (if $n \equiv 3,4$ ) with $n \bmod 6$. Then $d_{n+1}^{(1,4)}=2 d_{n}^{(1,4)}-d_{n-2}^{(1,4)}+\left(f_{n-8}^{(4)}+\lambda_{n}\right)$.

Proof. Write $d_{n}^{(1,4)}=d_{n}$. Then $\left\{d_{n}\right\}_{n \geq 1}=\{0,1,2,4,6,10,17,29,49,81, \cdots\}$, so when $n=9, \lambda_{9}=-1$ and $2 d_{9}-d_{7}+f_{1}^{(4)}+\lambda_{9}=81=d_{10}$. We assume the identity $d_{k+1}=2 d_{k}-d_{k-2}+f_{k-8}^{(4)}+\lambda_{k}$ for $k<n$. Then by means of the induction hypothesis, we have

$$
\begin{aligned}
2 d_{n}-d_{n-2}+f_{n-8}^{(4)}+\lambda_{n}= & \left(4 d_{n-1}-4 d_{n-3}+d_{n-5}\right)+\left(2 f_{n-9}^{(4)}-f_{n-11}^{(4)}+f_{n-8}^{(4)}\right) \\
& +\left(2 \lambda_{n-1}-\lambda_{n-3}+\lambda_{n}\right) . \quad(a)
\end{aligned}
$$

On the other hand due to Lemma 1 we also have

$$
4 d_{n-1}-4 d_{n-3}+d_{n-5}=f_{n+1}^{(1)}-\left(4 f_{n-1}^{(4)}-4 f_{n-3}^{(4)}+f_{n-5}^{(4)}\right)
$$

Hence together with (a) and (b), we have

$$
\begin{aligned}
2 d_{n}-d_{n-2}+f_{n-8}^{(4)}+\lambda_{n}= & f_{n+1}^{(1)}-\left(4 f_{n-1}^{(4)}-4 f_{n-3}^{(4)}+f_{n-5}^{(4)}\right) \\
& +\left(2 f_{n-9}^{(4)}-f_{n-11}^{(4)}+f_{n-8}^{(4)}\right)+\left(2 \lambda_{n-1}-\lambda_{n-3}+\lambda_{n}\right) \\
= & f_{n+1}^{(1)}-\left(4 f_{n-1}^{(4)}-4 f_{n-3}^{(4)}+f_{n-5}^{(4)}-f_{n-8}^{(4)}-2 f_{n-9}^{(4)}+f_{n-11}^{(4)}\right) \\
& +\left(2 \lambda_{n-1}-\lambda_{n-3}+\lambda_{n}\right) .
\end{aligned}
$$

But since

$$
4 f_{n-1}^{(4)}-4 f_{n-3}^{(4)}+f_{n-5}^{(4)}-f_{n-8}^{(4)}-2 f_{n-9}^{(4)}+f_{n-11}^{(4)}=f_{n+1}^{(4)}+2 C_{n},
$$

by Theorem 11, it therefore follows that

$$
\begin{aligned}
2 d_{n}-d_{n-2}+f_{n-8}^{(4)}+\lambda_{n} & =f_{n+1}^{(1)}-f_{n+1}^{(4)}-2 C_{n}+\left(2 \lambda_{n-1}-\lambda_{n-3}+\lambda_{n}\right) \\
& =d_{n+1}-2 C_{n}+\left(\lambda_{n}+2 \lambda_{n-1}-\lambda_{n-3}\right) .
\end{aligned}
$$


If we can show $\lambda_{n}+2 \lambda_{n-1}-\lambda_{n-3}=2 C_{n}$ for all $n(\bmod 6)$ then it completes to prove $d_{n+1}^{(1,4)}=2 d_{n}^{(1,4)}-d_{n-2}^{(1,4)}+\left(f_{n-8}^{(4)}+\lambda_{n}\right)$. Indeed, if $n \equiv 0$ then $\lambda_{n}+2 \lambda_{n-1}-$ $\lambda_{n-3}=1+2 \cdot 0+1=2=2 C_{n}$, and if $n \equiv 1$ then $1+2 \cdot 1+1=4=2 C_{n}$, etc.

Corollary 13. For all $n, f_{n}^{(4)}=d_{n+4}^{(1,4)}-d_{n+3}^{(1,4)}-d_{n+2}^{(1,4)}-\lambda_{n+2}$.

Proof. Theorem 8 and 12 give rise to

$$
\begin{aligned}
d_{n+1}^{(1,4)} & =2 d_{n}^{(1,4)}-d_{n-2}^{(1,4)}+d_{n-4}^{(1,4)}-d_{n-5}^{(1,4)}-d_{n-6}^{(1,4)} \\
& =2 d_{n}^{(1,4)}-d_{n-2}^{(1,4)}+f_{n-8}^{(4)}+\lambda_{n},
\end{aligned}
$$

so $f_{n-8}^{(4)}+\lambda_{n}=d_{n-4}^{(1,4)}-d_{n-5}^{(1,4)}-d_{n-6}^{(1,4)}$ and $f_{n}^{(4)}=d_{n+4}^{(1,4)}-d_{n+3}^{(1,4)}-d_{n+2}^{(1,4)}-\lambda_{n+8}$. It finishes the proof since $\lambda_{n+8}=\lambda_{n+2}$.

Indeed a Fibonacci 4-number $f_{n}^{(4)}$ can be obtained from the relation that, for instance $d_{24}^{(1,4)}-d_{23}^{(1,4)}-d_{22}^{(1,4)}-\lambda_{22}=74594-46043-28412+1=140=f_{20}^{(4)}$.

\section{References}

[1] S. Dey, H. Qaheri, S. Sane, S. Sanyal, A note on the bounds for the generalized Fibonacci $p$-sequence and its application in data-hiding, International J. of Computer Science and application, 7 (2010), 1-15.

[2] E. Kilic, The Binet formula, sums and representations of generalized Fibonacci $p$ numbers, European J. of Combinatorics, 29 (2008), 701-711.

[3] E. Kilic, A. Stakhov, On the Fibonacci and Lucas p-numbers, their sums, families of bipartite graphs and permanents of certain matrices. Chaos, Solitons and Fractals, 40 (2009), 2210-2221.

[4] J.P. Allouche, J. Johnson, Narayanas cows and delayed morphisms, In: Articles of 3rd Computer Music Conference JIM96, France, (1996).

[5] A. Stakhov, A generalization of the Fibonacci Q-matrix. Rep Natl Acad Sci Ukraine, 9 (1999), 46-49.

[6] A. Stakhov, B. Rozin, Theory of Binet formulas for Fibonacci and Lucas p-numbers, Chaos, Solitons and Fractals, 27 (2006), 1162-1177. 